\title{
THERE ARE ASYMPTOTICALLY THE SAME NUMBER OF LATIN SQUARES OF EACH PARITY
}

\author{
NICHOLAS J. CAVENAGH and IAN M. WANLESS
}

\begin{abstract}
A Latin square is reduced if its first row and column are in natural order. For Latin squares of a particular order $n$ there are four possible different parities. We confirm a conjecture of Stones and Wanless by showing asymptotic equality between the numbers of reduced Latin squares of each possible parity as the order $n \rightarrow \infty$.
\end{abstract}

2010 Mathematics subject classification: 05B15.

Keywords and phrases: Latin square; parity; Alon-Tarsi conjecture; row cycle.

\section{Introduction}

The parity of permutations plays a fundamental role in group theory. Latin squares can be thought of as two dimensional permutations and they also have a notion of parity. A Latin square has three attributes each of which can be even or odd, although any two of these attributes determines the third. There are thus four different parities that Latin squares of a given order may have. These parities account, for example, for the fragmentation of switching graphs $[5,13]$ and the failure of certain topological biembeddings [9]. They can also assist in diagnosing symmetries of Latin squares [6].

Unlike what happens for permutation groups, there can be different numbers of Latin squares of each parity. This difference is central to a famous conjecture by AlonTarsi [1] which has ramifications well beyond its apparent scope [4, 8]. Nevertheless, numerical evidence $[5,12,13]$ suggests that within several natural classes of Latin squares there are very close to the same number of each parity. The present note and [2] are the first to prove parities are asymptotically equinumerous (although [12] did show a weaker result in this direction). An advantage of the present work over [2] is that we prove a non-trivial result for all orders, whilst [2] only applies to even orders.

A Latin square of order $n$ is an $n \times n$ array of $n$ symbols such that each symbol occurs exactly once in each row and exactly once in each column. We will take the symbol set to be $[n]:=\{1,2, \ldots, n\}$, matching the row and column indices. A Latin square is normalised if the first row is $(1,2, \ldots, n)$. A Latin square is reduced if the 


\begin{tabular}{|l|}
\hline If $n \equiv 0$ or $1(\bmod 4)$ \\
\hline$R_{n}^{\mathrm{E}}=R_{n}^{\mathrm{sE}}=R_{n}^{000}+R_{n}^{110}$ \\
$R_{n}^{\mathrm{O}}=R_{n}^{\mathrm{SO}}=R_{n}^{011}+R_{n}^{101}$ \\
\hline$U_{n}^{\mathrm{E}}=R_{n}^{\mathrm{CE}}=R_{n}^{000}+R_{n}^{101}$ \\
$U_{n}^{\mathrm{o}}=R_{n}^{\mathrm{CO}}=R_{n}^{011}+R_{n}^{110}$ \\
\hline$R_{n}^{\mathrm{RE}}=R_{n}^{000}+R_{n}^{011}=U_{n}^{\mathrm{E}}$ \\
$R_{n}^{\mathrm{RO}}=R_{n}^{101}+R_{n}^{110}=U_{n}^{\mathrm{o}}$ \\
\hline$R_{n}^{111}=R_{n}^{100}=R_{n}^{010}=R_{n}^{001}=0$ \\
$R_{n}^{011}=R_{n}^{101}$ \\
$R_{n}^{011}=R_{n}^{101}=R_{n}^{110}$ when $n$ is even \\
\hline
\end{tabular}

\begin{tabular}{|l|}
\hline If $n \equiv 2$ or $3(\bmod 4)$ \\
\hline$R_{n}^{\mathrm{E}}=R_{n}^{\mathrm{so}}=R_{n}^{111}+R_{n}^{001}$ \\
$R_{n}^{\mathrm{O}}=R_{n}^{\mathrm{SE}}=R_{n}^{100}+R_{n}^{010}$ \\
\hline$U_{n}^{\mathrm{E}}=R_{n}^{\mathrm{CO}}=R_{n}^{111}+R_{n}^{010}$ \\
$U_{n}^{\mathrm{o}}=R_{n}^{\mathrm{CE}}=R_{n}^{100}+R_{n}^{001}$ \\
\hline$R_{n}^{\mathrm{RO}}=R_{n}^{111}+R_{n}^{100}=U_{n}^{\mathrm{E}}$ \\
$R_{n}^{\mathrm{RE}}=R_{n}^{010}+R_{n}^{001}=U_{n}^{\mathrm{o}}$ \\
\hline$R_{n}^{000}=R_{n}^{011}=R_{n}^{101}=R_{n}^{110}=0$ \\
$R_{n}^{100}=R_{n}^{010}$ \\
$R_{n}^{100}=R_{n}^{010}=R_{n}^{001}$ when $n$ is even \\
\hline
\end{tabular}

TABLE 1. Table of identities.

first row is $(1,2, \ldots, n)$ and the first column is $(1,2, \ldots, n)^{T}$. A Latin square $L=\left(l_{i j}\right)$ is unipotent if $l_{11}=l_{22}=\cdots=l_{n n}$.

Suppose $P$ is a property of Latin squares of order $n$. Let $L_{n}^{P}, R_{n}^{P}$ and $U_{n}^{P}$ be the numbers respectively of Latin squares, reduced Latin squares and normalised unipotent Latin squares of order $n$ with property $P$. If $P$ is omitted we count the whole class.

Let $\mathcal{S}_{n}$ denote the permutations of $[n]$ and $\zeta: \mathcal{S}_{n} \mapsto \mathbb{Z}_{2}$ the usual sign homomorphism with kernel the alternating group. Given a Latin square $L=\left(l_{i j}\right)$ of order $n$, we can identify the following $3 n$ permutations in $\mathcal{S}_{n}$. For all $i \in[n]$ define $\sigma_{i}^{\text {row }}$ by $\sigma_{i}^{\text {row }}(j)=l_{i j}$. For all $j \in[n]$ define $\sigma_{j}^{\text {col }}$ by $\sigma_{j}^{\text {col }}(i)=l_{i j}$. For all $\ell \in[n]$ define $\sigma_{\ell}^{\text {sym }}$ such that $\sigma_{\ell}^{\text {sym }}(i)$ is equal to the $j$ for which $l_{i j}=\ell$. We call $\pi_{\text {row }}:=\sum_{i} \zeta\left(\sigma_{i}^{\text {row }}\right)$, $\pi_{\mathrm{col}}:=\sum_{j} \zeta\left(\sigma_{j}^{\mathrm{col}}\right)$ and $\pi_{\mathrm{sym}}:=\sum_{\ell} \zeta\left(\sigma_{\ell}^{\mathrm{sym}}\right)$ the row-parity, column-parity and symbolparity of $L$, respectively. A Latin square is called even or odd if $\pi_{\text {row }}+\pi_{\text {col }} \equiv 0$ or $1 \bmod 2$, respectively. A Latin square is called row-even or row-odd if $\pi_{\text {row }} \equiv 0$ or 1 , respectively. A Latin square is called column-even or column-odd if $\pi_{\mathrm{col}} \equiv 0$ or 1 , respectively. A Latin square is called symbol-even or symbol-odd if $\pi_{\text {sym }} \equiv 0$ or 1 , respectively. We define the properties:

$\begin{array}{llll}\mathrm{E} & =\text { "is an even Latin square" } & \mathrm{CE} & =\text { "is a column-even Latin square" } \\ \mathrm{O} & =\text { "is an odd Latin square" } & \text { CO } & =\text { "is a column-odd Latin square" } \\ \mathrm{RE} & =\text { "is a row-even Latin square" } & \text { SE } & =\text { "is a symbol-even Latin square" } \\ \mathrm{RO} & =\text { "is a row-odd Latin square" } & \text { SO } & =\text { "is a symbol-odd Latin square" }\end{array}$

We define the parity of a Latin square $L$ to be the ordered triple $\pi=\pi_{\text {row }} \pi_{\text {col }} \pi_{\text {sym }}$. Writing $\pi$ as a superscript denotes that we are restricting to Latin squares with parity $\pi$. Some of the basic relationships that are proved in [12] are summarised in Table 1. 
We use standard ' $\sim$ ', 'little- $O$ ', 'big- $O$ ' and 'big- $\Omega$ ' asymptotic notation, always with the order of our Latin squares $n \rightarrow \infty$. The aim of this note is to confirm a conjecture from [12] by proving the following result.

Theorem 1. As $n \rightarrow \infty$,

$$
\begin{aligned}
& \left.\begin{array}{l}
L_{n}^{000} \sim L_{n}^{011} \sim L_{n}^{101} \sim L_{n}^{110} \sim \frac{1}{4} L_{n} \\
R_{n}^{000} \sim R_{n}^{011} \sim R_{n}^{101} \sim R_{n}^{110} \sim \frac{1}{4} R_{n} \\
U_{n}^{000} \sim U_{n}^{011} \sim U_{n}^{101} \sim U_{n}^{110} \sim \frac{1}{4} U_{n}
\end{array}\right\} \text { for } n \equiv 0,1 \quad(\bmod 4), \\
& \left.\begin{array}{l}
L_{n}^{111} \sim L_{n}^{100} \sim L_{n}^{010} \sim L_{n}^{001} \sim \frac{1}{4} L_{n} \\
R_{n}^{111} \sim R_{n}^{100} \sim R_{n}^{010} \sim R_{n}^{001} \sim \frac{1}{4} R_{n} \\
U_{n}^{111} \sim U_{n}^{100} \sim U_{n}^{010} \sim U_{n}^{001} \sim \frac{1}{4} U_{n}
\end{array}\right\} \text { for } n \equiv 2,3 \quad(\bmod 4), \\
& L_{n}^{\mathrm{E}} \sim L_{n}^{\mathrm{O}} \sim L_{n}^{\mathrm{RE}} \sim L_{n}^{\mathrm{RO}} \sim L_{n}^{\mathrm{CE}} \sim L_{n}^{\mathrm{CO}} \sim L_{n}^{\mathrm{SE}} \sim L_{n}^{\mathrm{So}} \sim \frac{1}{2} L_{n}, \\
& R_{n}^{\mathrm{E}} \sim R_{n}^{\mathrm{O}} \sim R_{n}^{\mathrm{RE}} \sim R_{n}^{\mathrm{RO}} \sim R_{n}^{\mathrm{CE}} \sim R_{n}^{\mathrm{CO}} \sim R_{n}^{\mathrm{SE}} \sim R_{n}^{\mathrm{SO}} \sim \frac{1}{2} R_{n}, \\
& U_{n}^{\mathrm{E}} \sim U_{n}^{\mathrm{o}} \sim U_{n}^{\mathrm{RE}} \sim U_{n}^{\mathrm{RO}} \sim U_{n}^{\mathrm{cE}} \sim U_{n}^{\mathrm{co}} \sim U_{n}^{\mathrm{SE}} \sim U_{n}^{\mathrm{sO}} \sim \frac{1}{2} U_{n} .
\end{aligned}
$$

In contrast, the Alon-Tarsi conjecture [1] asserts that $L_{n}^{\mathrm{E}} \neq L_{n}^{\mathrm{O}}$ for even $n$. Two distinct generalisations of this are by Zappa [15], who suggests that $U_{n}^{\mathrm{E}} \neq U_{n}^{\mathrm{o}}$ for all $n$ and Stones and Wanless [12] who suggest that $R_{n}^{\mathrm{E}} \neq R_{n}^{\mathrm{o}}$ for all $n$. These conjectures are only known to be true in some very special cases (see $[7,11,12]$ for details).

There is a natural action of $\mathcal{S}_{n} \times \mathcal{S}_{n} \times \mathcal{S}_{n}$ on Latin squares called isotopism. Its orbits are called isotopism classes. In essence, the reason that the Alon-Tarsi conjecture is restricted to even orders is that parity is an isotopism class invariant for even orders but not for odd orders. Since it is known that asymptotically almost all Latin squares have trivial stabiliser in the group of isotopisms [10], a corollary of Theorem 1 is that for even $n \rightarrow \infty$ there are asymptotically equal numbers of isotopism classes of Latin squares of each of the possible parities.

\section{Parities are equinumerous}

Whenever we use the word "random" it will be implicit that we are referring to the discrete uniform distribution (technically, actually a sequence of such distributions as $n \rightarrow \infty)$.

A row cycle of length $\ell$ is a minimal (in the sense of containment) non-empty $2 \times \ell$ submatrix of a Latin square such that each row of the submatrix contains the same symbols. We say that a row cycle is even or odd depending on whether its length $\ell$ is even or odd, respectively. The two rows within a row cycle can be switched to give a slightly different Latin square. By switching an odd row cycle we change the column parity and the symbol parity, while leaving the row parity unchanged [13]. This simple observation will be the key to our result. 
Our aim is to find an odd row cycle that does not meet the first row or first column. We want to show that a random reduced Latin square can be expected to have such a cycle. However, we do this by first showing that a random Latin square also has such a cycle. This allows us to employ techniques that may move beyond the set of reduced Latin squares. The techniques in question were developed in [3] to study row cycle lengths in a random Latin square. It will suit us to adapt the results of [3], which dealt with the first two rows, to the last two rows instead. Similarly, [3] allowed conditioning on the contents of a set $F$ of columns. In that paper $F$ was the last $n-m$ columns, however it will suit us to use a variable set of columns which includes the first column. The results from [3] apply unchanged, given the symmetry between different columns, and between different rows.

We will consider random Latin squares of order $n$ as $n \rightarrow \infty$. Soon we will want to consider probabilities that are conditional on our Latin square including a set $F$ of $n-m$ columns that includes the first column. A prerequisite for the methods of [3] is that $F$ must contain entire row cycles in its last two rows. We impose the extra condition that $F$ contains a single row cycle in its last two rows. With this assumption it turns out that $F$ is unlikely to be too big:

Lemma 2. With probability 1 -o(1) a random Latin square of order $n$ has no cycle longer than $n-\log n$ within the last two rows.

Proof. Let $p$ be the probability that a random permutation in $\mathcal{S}_{n}$ has a cycle of length at least $n-\log n$. Then

$$
p=\frac{1}{n !} \sum_{i=\lceil n-\log n\rceil}^{n}\left(\begin{array}{l}
n \\
i
\end{array}\right)(i-1) !(n-i) !=\sum_{i=\lceil n-\log n\rceil}^{n} \frac{1}{i}=O\left(\frac{\log n}{n}\right) .
$$

Let $\xi$ be the multiset of the lengths of the row cycles in the last two rows of a random Latin square of order $n$. If $\xi$ has an element of size at least $n-\log n$, then $\xi$ has at most $(\log n) / 2+1$ elements. Hence by (1) and [3, Cor. 4.5], the probability that $\xi$ has an element of size at least $n-\log n$ is at most $n^{1 / 3} 2^{(\log n) / 2+1} p=o\left(n^{-0.3}\right)$.

As foreshadowed, we now wish to condition on a random Latin square $L$ containing a set $F$ of entries consisting of entire columns (including the first), where in the last two rows the entries of $F$ form a single row cycle. This framework is consistent with [3]. Let $m$ be the number of columns that are not in $F$. Let $\rho$ be the partition of $m$ formed by the lengths of the row cycles in the last two rows that are not in $F$. We will consider $m$ and $\rho$ to be discrete random variables in the resulting probability space. Our results will be phrased in terms of $m$ and $\rho$ but are otherwise independent of $F$. Note that with high probability $m \rightarrow \infty$ as $n \rightarrow \infty$, by Lemma 2. From [3, Thm 4.9], we have:

Lemma 3. There exists a constant $c$ with $0<c<1$ such that $\rho$ has fewer than $9 \sqrt{m}$ parts with probability $1-o\left(c^{m}\right)$. 
Let $P(m)$ denote the partitions of $m$ into parts of size at least 2. Let

$$
\gamma(\lambda)=\frac{m !}{\prod_{i=2}^{m} \lambda_{i} ! i^{\lambda_{i}}}
$$

be the number of derangements with cycle structure $\lambda=\left(2^{\lambda_{2}}, 3^{\lambda_{3}}, \ldots, m^{\lambda_{m}}\right) \in P(m)$. Here and henceforth, $i^{\lambda_{i}}$ denotes $\lambda_{i}$ parts of size $i$, where we allow the possibility that $\lambda_{i}=0$. Let $S(\lambda, F)$ denote the set of Latin squares that contain $F$ and have $\rho=\lambda$.

Lemma 4. Let $m$ be even and suppose that $\lambda=\left(2^{\lambda_{2}}, 4^{\lambda_{4}}, \ldots, m^{\lambda_{m}}\right) \in P(m)$ has only even parts, including one of size $z$ where $z \rightarrow \infty$ as $n \rightarrow \infty$. Let $M$ be the set of $\mu \in P(m)$ such that $\mu$ is obtained from $\lambda$ by splitting a part of size $z$ into two parts of odd size. Then

$$
\sum_{\mu \in M}|S(\mu, F)|=|S(\lambda, F)| \Omega(\log z) .
$$

Proof. Let $\mu=\left(2^{\mu_{2}}, 3^{\mu_{3}}, \ldots, m^{\mu_{m}}\right) \in M$ be such that $\mu$ is obtained from $\lambda$ by splitting one part of size $z$ into parts of size $a$ and $z-a$, where $a$ is odd (and thus $z-a$ is too) and $a<z-a$. Since $\lambda$ has only even parts, $\lambda_{a}=\lambda_{z-a}=0$ and $\mu_{a}=\mu_{z-a}=1$. Moreover $\lambda_{z}=\mu_{z}+1 \geqslant 1$. By (2),

$$
\frac{\gamma(\mu)}{\gamma(\lambda)}=\frac{z \lambda_{z}}{a(z-a)}
$$

By [3, Lem 3.13], this implies that

$$
|S(\mu, F)| \geqslant \frac{2 z \lambda_{z}|S(\lambda, F)|}{3 a(z-a)} \geqslant \frac{2 z|S(\lambda, F)|}{3 a(z-a)} .
$$

Thus,

$$
\sum_{\mu \in M}|S(\mu, F)| \geqslant \frac{2}{3}|S(\lambda, F)| z \sum_{a=1}^{w} \frac{1}{(2 a+1)(z-2 a-1)},
$$

where $w=\lfloor(z-3) / 4\rfloor$ is the largest integer satisfying $2 w+1<z-2 w-1$. However, $1 /((2 x+1)(z-2 x-1))$, is a decreasing function of $x$ for $1 \leqslant x \leqslant w$ so

$$
\begin{aligned}
\sum_{a=1}^{w} \frac{1}{(2 a+1)(z-(2 a+1))} & \geqslant \int_{1}^{w} \frac{d x}{(2 x+1)(z-2 x-1)} \\
& =\frac{1}{2 z} \log \frac{(2 w+1)(z-3)}{3(z-2 w-1)}=\Omega\left(\frac{\log z}{z}\right),
\end{aligned}
$$

from which the result follows.

We next show that with high probability there is an odd cycle that does not meet the first row or column (assuming $n>2$ ). We deduce this first for general Latin squares, then infer it for reduced Latin squares. 
Theorem 5. With probability 1 - o(1) there is a part of odd size in $\rho$.

Proof. By Lemma 2 we know that $m \geqslant \log n$ with probability $1-o(1)$. By Lemma 3, there asymptotically almost surely are at most $9 \sqrt{m}$ parts in $\rho$, so there is some part of size at least $\sqrt{m} / 9$. By Lemma 4 , the probability of $\rho$ having no odd parts is at most $O(1 / \log m)=o(1)$, as claimed.

Corollary 6. With probability $1-o(1)$, in the last two rows of a random reduced Latin square of order $n$ there is a cycle of odd length that does not include the first column.

Proof. We can reduce a Latin square by permuting the symbols so that the first column is in order, then permuting the columns so that the first row is in order. These operations do not affect whether the last two rows contain a cycle of odd length that does not include the first column (note that the first column does not move). Also, each reduced Latin square is produced the same number of times, namely $n !(n-1)$ ! times, when the above reduction is applied to all Latin squares. So reduced Latin squares have the same probability of having the property of interest as general Latin squares do.

We are now in a position to prove our main result. As already noted, by switching an odd row cycle we change the column parity and the symbol parity. Hence Corollary 6 provides us with an involution, which acts on all but a negligible fraction of reduced Latin squares, and which reverses column parity and reverses symbol parity. It follows that $R_{n}^{\mathrm{CE}} \sim R_{n}^{\mathrm{co}}$ and $R_{n}^{\mathrm{SE}} \sim R_{n}^{\mathrm{so}}$. Table 1 then tells us that,

$$
\begin{array}{lll}
R_{n}^{000} \sim R_{n}^{011} \sim R_{n}^{101} \sim R_{n}^{110} \sim \frac{1}{4} R_{n} & \text { for } n \equiv 0,1 \quad(\bmod 4), \\
R_{n}^{111} \sim R_{n}^{100} \sim R_{n}^{010} \sim R_{n}^{001} \sim \frac{1}{4} R_{n} & \text { for } n \equiv 2,3 \quad(\bmod 4),
\end{array}
$$

as $n \rightarrow \infty$. The remainder of Theorem 1 can then be easily deduced from Table 1 and the following additional observations. Replacing each row of a Latin square by its inverse (when considered as a permutation) converts reduced Latin squares into normalised unipotent Latin squares and vice versa. Hence $R_{n}^{a b c}=U_{n}^{a c b}$ for all parities $\pi=a b c$ and all $n$. We also know two more facts from [12]. Firstly, for even $n$,

$$
L_{n}^{P}=n !(n-1) ! R_{n}^{P}=n !(n-1) ! U_{n}^{P},
$$

whenever $P \in\{\mathrm{E}, \mathrm{O}, \mathrm{RE}, \mathrm{RO}, \mathrm{CE}, \mathrm{CO}, \mathrm{SE}, \mathrm{so}\}$ or $P$ is any parity. Secondly, for odd $n \geqslant 3$,

$$
L_{n}^{000}=L_{n}^{011}=L_{n}^{101}=L_{n}^{110} \text { and } L_{n}^{111}=L_{n}^{100}=L_{n}^{010}=L_{n}^{001} .
$$

\section{Concluding comments}

We have confirmed a conjecture from [12] and explained why the large components have comparable size in the switching graphs studied in [13]. Our results do not explain why the components in the switching graphs in [5] have comparable size. At this stage we have no tools to study the lengths of cycles in random symmetric Latin squares. 
A much stronger result than Theorem 1 seems very likely to be true. By Wilf [14, p.209], the proportion of permutations in $\mathcal{S}_{n}$ that have no odd cycles is $2^{-n} n ! /(n / 2) !^{2} \sim$ $\sqrt{2 /(\pi n)}$. It follows immediately that the proportion of derangements with no odd cycles is also $O\left(n^{-1 / 2}\right)$. Hence the proportion of derangements with at most one odd cycle is $O\left(n^{-1 / 2}\right)$ if $n$ is even and no more than

$\frac{(n-1) !}{n !}+\frac{O(1)}{n !} \sum_{i=0}^{(n-3) / 2}\left(\begin{array}{c}n \\ 2 i+1\end{array}\right) \frac{(2 i) !(n-2 i-1) !}{(n-2 i-1)^{1 / 2}}=\frac{1}{n}+O(1) \sum_{i=0}^{(n-3) / 2} \frac{1}{(2 i+1)(n-2 i-1)^{1 / 2}}$

if $n$ is odd. Approximating the sum by an integral, we find that for all $n$ the proportion of derangements with at most one odd cycle is $O\left(n^{-1 / 2} \log n\right)$. If [3, Conj. 6.1] holds then a similar statement would be true about the cycles in the last two rows of a Latin square: namely there would be at least two odd cycles with probability $1-O\left(n^{-1 / 2} \log n\right)$. At least one of these cycles is switchable in the sense that it does not hit the first column. Amongst the squares with no switchable odd cycle in the last two rows, we can look for a switchable odd cycle in rows $n-3, n-2$, then in rows $n-5, n-4$ and so on up to, but not including, the first row. Switching the first switchable cycle that we find in this way would give us an involution, because switching cycles in rows $x$ and $y$ never affects the cycle lengths between rows other than $x$ and $y$. The domain of the involution includes all reduced Latin squares that have any switchable cycle in an appropriate pair of rows. It seems plausible that each pair of rows would have a switchable cycle with something close to an independent probability $1-O\left(n^{-1 / 2} \log n\right)$, meaning the proportion of reduced Latin squares outside the domain of our involution would be $O\left(n^{-c n}\right)$ for some constant $c>0$. Hence for each given $n$ the numbers of reduced Latin squares with each of the four possible parities are probably very much closer to each other than our work has demonstrated.

\section{References}

[1] N. Alon and M. Tarsi, Colorings and orientations of graphs, Combinatorica 12 (1992) 125-134.

[2] L. Alpoge, Square-root cancellation for the signs of Latin squares, Combinatorica, to appear. DOI: 10.1007/s00493-015-3373-7.

[3] N. J. Cavenagh, C. Greenhill, and I. M. Wanless, The cycle structure of two rows in a random Latin square, Random Structures Algorithms, 33 (2008) 286-309.

[4] R. Huang and G.-C. Rota, On the relations of various conjectures on Latin squares and straightening coefficients, Discrete Math. 128 (1994), 225-236.

[5] P. Kaski, A. D. S. Medeiros, P. R. J. Östergård and I. M. Wanless, Switching in one-factorisations of complete graphs, Electron. J. Comb. 21(2) (2014), \#P2.49.

[6] D. Kotlar, Parity types, cycle structures and autotopisms of Latin squares, Electron. J. Combin. 19(3) (2012), \#P10, 17 pp.

[7] D. Kotlar, On extensions of the Alon-Tarsi Latin square conjecture, Electron. J. Combin. 19(4) (2012), \#P7, 10 pp.

[8] S. Kumar and J. M. Landsberg, Connections between conjectures of Alon-Tarsi, Hadamard-Howe, and integrals over the special unitary group, Discrete Math. 338 (2015), 1232-1238. 
[9] J. G. Lefevre, D. M. Donovan, M. J. Grannell and T. S. Griggs, A constraint on the biembedding of Latin squares, European J. Combin. 30 (2009), 380-386.

[10] B. D. McKay and I. M. Wanless, On the number of Latin squares, Ann. Comb. 9 (2005), 335-344.

[11] D. S. Stones, Formulae for the Alon-Tarsi conjecture, SIAM J. Discrete Math. 26 (2012), 65-70.

[12] D. S. Stones and I. M. Wanless, How not to prove the Alon-Tarsi conjecture, Nagoya Math. J. 205 (2012), 1-24.

[13] I. M. Wanless, Cycle switches in Latin squares, Graphs Combin. 20 (2004) 545-570.

[14] H.S. Wilf, Generatingfunctionology (2nd ed.), Academic Press, San Diego, 1994.

[15] P. Zappa, The Cayley determinant of the determinant tensor and the Alon-Tarsi Conjecture, Adv. in Appl. Math. 13 (1997) 31-44.

Nicholas J. Cavenagh, Department of Mathematics, University of Waikato, Private Bag 3105, Hamilton, New Zealand.

e-mail: nickc@waikato.ac.nz

Ian M. Wanless, School of Mathematical Sciences, Monash University, Vic 3800, Australia.

e-mail: ian.wanless@monash.edu 